\title{
ADVERSE EFFECT OF INSOMNIA ON BLOOD LIPIDS IN PATIENTS WITH HYPERTENSION
}

\author{
Anna Isayeva \\ Department of Complex risk Reduction of Chronic non-communicable diseases ${ }^{1}$ \\ anna_isayeva_74@yahoo.co.uk \\ Olena Buriakovska \\ Department of Complex risk Reduction of Chronic non-communicable diseases ${ }^{I}$ \\ alena.tbtch@gmail.com \\ Oleksander Martynenko \\ Department of Hygiene and Social Medicine, School of Medicine ${ }^{2}$ \\ alexander.v.martynenko@karazin.ua \\ Sergiy Ostropolets \\ Department of Hygiene and Social Medicine, School of Medicine ${ }^{2}$ \\ s.ostropolets@karazin.ua \\ ${ }^{1}$ The Government Institution «National Institute of Therapy \\ named by L. T. Malaya of National Ukrainian Academy of Medical Science» \\ 2 a, Lyubovi Maloy ave., Kharkiv, Ukraine, 61039 \\ ${ }^{2}$ V. N. Karazin Kharkiv National University \\ 4 Svobody sq., Kharkiv, Ukraine, 61022
}

\begin{abstract}
Insomnia is a risk factor for the development of arterial hypertension, obesity, type 2 diabetes mellitus, cardiac rhythm disorders, and myocardial infarction. At the same time, insomnia is one of the most frequent non-cardiac complaints in patients with cardiovascular diseases.

The aim of the work was to study the presence of possible relationships between insomnia and the level of blood lipids.

Materials and methods. A cross-sectional study involving 118 patients was conducted. Criteria for inclusion in the study were age over 45 years, the presence of essential hypertension. All patients included the study underwent sampling of $7 \mathrm{ml}$ of venous blood in the morning under fasting conditions. The content of total cholesterol (TCS), triglycerides (TG), high-density lipoprotein cholesterol (HDL CS) was determined by enzymatic method on a biochemical analyser Humalyzer 2000. The patient was interviewed by a pre-trained study doctor.

Results. In the article a relationship between total cholesterol, low-density lipoprotein cholesterol and the presence of insomnia has been established and proved by statistical model. The overall statistical model accuracy is $89.6 \%$ and statistical significance $p<0.005$. Accuracy of insomnia prediction is $85.7 \%$ by level of total cholesterol (TCS) and patient interview data. Only one model with best accuracy exists and it was estimated at the article.

Conclusions. Relationship between total cholesterol, low-density lipoprotein cholesterol and the presence of insomnia has been established and proved by statistical model. Accuracy of insomnia prediction is $85.7 \%$ by level of total cholesterol (TCS) and patient interview data.
\end{abstract}

Keywords: insomnia, hypertension, cholesterol, sleep disorders, low-density lipoprotein cholesterol.

DOI: $10.21303 / 2504-5679.2021 .001600$

\section{Introduction}

Insomnia is a risk factor for the development of arterial hypertension, obesity, type 2 diabetes mellitus, cardiac rhythm disorders, and myocardial infarction [1]. At the same time, insomnia is one of the most frequent non-cardiac complaints in patients with cardiovascular diseases. More than $40 \%$ of patients with cardiovascular diseases had some manifestations of insomnia [2]. For comparison, insomnia is diagnosed in no more than $10-13 \%$ of individuals in the general population [3]. At the same time, studies on the relationship between insomnia and indicators of blood lipids demonstrate 
quite contradictory results. An association has been established between the duration of sleep and HDL cholesterol level. Thus, according to another authors patients who sleep less than 6 hours and more than 8 hours, have low HDL cholesterol levels [4, 5]. It was established a link between short duration of sleep and low HDL cholesterol level [6]. It was demonstrated that improved sleep in patients with cardiovascular diseases was accompanied by a decrease in LDL cholesterol from 2.29 to $1.43 \mathrm{mmol} / 1$ [2].

Insomnia, according to the International Classification of Disorders (Issue 3), developed by the International Academy of Disorders in 2014, has to include various manifestations: difficulty initiating sleep or difficulty maintaining continuous sleep, daytime manifestations of sleep disturbances (behavioural problems, concentration on sleep). An indispensable condition should be the presence of these manifestations, despite the fact that the patient has adequate conditions for sleep. All of these components of insomnia can affect cholesterol metabolism in different ways. Thus, it has been established that cholesterol metabolism depends on the circadian cycle [7], and accordingly, insomnia can negatively affect blood lipid levels. In addition, insomnia is often associated with eating disorders, namely, late meals and nightly meals, which also negatively affects lipid metabolism $[6,8]$. One of the factors contributing to the development of lipid metabolism disorders is the increased activity of the sympathetic nervous system, usually associated with insomnia [9]. The negative effect of excessive sympathetic activity on blood lipids was demonstrated in some works [10].

The aim of the work was to study the presence of possible relationships between insomnia and the level of blood lipids.

\section{Materials and methods}

A cross-sectional study involving 118 patients was conducted. The study was conducted at the Government Institution «National Institute of Therapy named by L. T. Malaya of National Ukrainian Academy of Medical Science» in 2018-2019. Criteria for inclusion in the study were age over 45 years, the presence of essential hypertension. The diagnosis of essential hypertension was established in accordance with the recommendations for the management of patients with arterial hypertension of the European Society of Cardiology/European Society of Hypertension in 2018 [11]. The study did not involve patients with a history of cardiovascular events (myocardial infarction, stroke), patients with heart failure or ejection fraction less than $45 \%$, with persistent arterial hypertension, thyroid dysfunction, gastric ulcer and duodenal ulcer at exacerbation stage, with concomitant diseases that affect the quality of life, patients with degree 3 obesity, with concomitant active cancer diseases, with depressive states (organic depressive disorder (F 06.32), recurrent depressive disorder (F 33.0-3) or depressive episode of any severity (F 31.3-5, F 32.0-3), as well as mixed anxiety and depressive disorder (F 41.2) according to the criteria of the International Classification of Diseases, 10th revision (ICD-10), with insomnia as a symptom of any mental illness, with concomitant therapy with glucocorticosteroids, centrally acting antihypertensive drugs (moxonidine, reserpine), $\beta$-adrenergic blocking agents, somniferous agents, patients who confirmed consumption of food at night, patients with obstructive sleep apnoea syndrome.

\subsection{Biochemical testing}

All patients included in the study underwent sampling of $7 \mathrm{ml}$ of venous blood in the morning under fasting conditions, after 9 hours without food, from the cubital vein to measure the lipid spectrum. The blood was centrifuged at $3000 \mathrm{rpm}$ for 15 minutes. Plasma was used for further analysis, which was collected into a clean test tube.

The content of total cholesterol (TCS), triglycerides (TG), high-density lipoprotein cholesterol (HDL CS) was determined by enzymatic method on a biochemical analyzer Humalyzer 2000, No. 18300-5397 (Germany). Very low density lipoprotein cholesterol (VLDL CS) was calculated as the ratio of TG to factor 5. Low density lipoprotein cholesterol (LDL cholesterol) was calculated using the formula by W. T. Friedewald:

$$
\text { VLDL CS }=\text { TCS }-(\text { HDL CS+TG/2.22), }
$$

where TG/2.22 conforms to cholesterol concentration in the composition of VLDL [7]. 
Blood glucose level was measured by glucose oxidase method on a biochemical analyzer Humalyzer 2000, No. 18300-5397, the level of glycated hemoglobin (HbA1c) was measured by ion exchange method on the same analyzer.

\subsection{Assessment of the presence of insomnia}

The presence of insomnia was assessed using the criteria of the International Classification of Sleep Disorders (Issue 3), which was developed by the International Academy of Sleep Disorders in 2014: 1 - difficulty initiating sleep or maintaining continuous sleep; 2 - the availability of adequate sleeping conditions; 3 - daytime effects of poor sleep quality. The patient was interviewed by a pre-trained study doctor.

\subsection{Exclusion of sleep apnoea syndrome}

To detect sleep apnoea syndrome, night monitoring was performed using the SOMNOcheck micro CARDIO cardio-respiratory complex (Germany). The device allows to register the flow of breath, blood saturation, heart rate and is certified for the diagnosis of sleep apnoea syndrome.

\subsection{Ethical aspects}

The study was approved by the Committee on Ethics and Deontology of the State Institution «L. T. Malaya Therapy National Institute of the National Academy of Medical Sciences of Ukraine» on 28 April 2017. All participants of the study signed an informed consent; preliminarily they were clearly explained the purpose and objectives of the study.

\subsection{Statistical analysis}

The type of data distribution was checked by the Kolmogorov-Smirnov (K-S) test. With normal distribution of continuous values, the data are presented as mean values (M) and standard deviation (SD); for quantitative values, the data are presented as the number (n) and frequency (\%). The data that did not correspond to the normal distribution were presented as medians and $25 \%$ and $75 \%$ of quartiles. When comparing more than two groups, a one-way ANOVA test was used. When comparing two independent samples corresponding to the normal distribution, Student's $t$-test was used. If the independent samples did not correspond to the normal data distribution, comparisons were performed using the Mann-Whitney (M-W) test. Discrepancies were considered statistically significant at $p<0.05$.

To search and justify the relationship of the «Insomnia» variable with the measured values of cholesterol content (5 values) and history data (17 values), generalized linear mixed models (GLMM) were used. This type of model provides high flexibility in the construction and study of new hypotheses, since relationships are sought at the level of average values of variables, their variances and covariances. GLMM is implemented as the corresponding procedure of the IBM SPSS Statistics package.

\section{Results}

Table 1 provides clinical characterization of the examined population.

At the time of inclusion in the study, metformin was taken by all patients with diabetes mellitus in both groups. Angiotensin-converting enzyme (ACE) inhibitors were taken by 22 (45.8\%) patients in the group without symptoms of insomnia, and by $22(31.4 \%)$ patients in the group with symptoms of insomnia $\left(\chi^{2}=0.038 ; p=0.12\right)$. Sartans were taken by $31(44.2 \%)$ patients without symptoms of insomnia and $25(52.1 \%)$ patients with symptoms of insomnia $\left(\chi^{2}=0.69 ; p=0.45\right)$, indapamide - by $5(7.1 \%)$ and $3(6.3 \%)$ patients, respectively $\left(\chi^{2}=0.01 ; p=0.92\right)\left(\chi^{2}=0.02 ; p=0.58\right)$, torasemide - by $11(15.7 \%)$ and $8(16.7 \%)$ patients, respectively $\left(\chi^{2}=0.02 ; p=0.89\right)$. Patients in both groups received atorvastatin to monitor total cholesterol levels. The groups were comparable in terms of therapy.

The selection criteria for a statistically justified qualitative model are: information criteria (Akaike and Bayes) and the statistical significance of the model as a whole and the model variables (in ourcase, $p<0.05$ ). Thus, we tested all possible hypotheses and combinations of variables until 
a statistically significant model and all its independent variables were found. The fixed (main) effects are represented by variables: cholesterols - TCS; history variables - «Difficulty initiating sleep»; «Difficulty maintaining continuous sleep» and «Concentration on poor sleep». They all had a level of statistical significance $p<0.005$ (Fig. 1).

Table 1

General clinical characteristics of the examined patients with hypertension depending on the presence of insomnia symptoms $(\mathrm{M} \pm \mathrm{SD})$

\begin{tabular}{|c|c|c|c|c|}
\hline \multicolumn{2}{|c|}{ Parameter } & With insomnia symptoms $(n=48)$ & Without insomnia symptoms $(n=70)$ & $\chi^{2} ; p$ \\
\hline \multicolumn{2}{|l|}{ Age, years } & $57.9 \pm 9.8$ & $58.2 \pm 10.1$ & 0.46 \\
\hline \multicolumn{2}{|l|}{ Men } & $11(22.9 \%)$ & $16(22.8 \%)$ & 0.29 \\
\hline \multicolumn{2}{|c|}{ Diabetes mellitus type 2} & $32(66.7 \%)$ & $40(57.1 \%)$ & $1.07 ; 0.59$ \\
\hline \multicolumn{2}{|l|}{ Smoking } & $8(16.7 \%)$ & $11(15.7 \%)$ & $0.02 ; 0.89$ \\
\hline \multicolumn{2}{|c|}{ Regular physical exercise } & $9(18.8 \%)$ & $11(15.7 \%)$ & $0.19 ; 0.66$ \\
\hline \multicolumn{2}{|l|}{ SBP, $\mathrm{mm} \mathrm{Hg}$} & $138.2 \pm 12.8$ & $130.0 \pm 5.3$ & 0.12 \\
\hline \multicolumn{2}{|l|}{$\mathrm{DBP}, \mathrm{mm} \mathrm{Hg}$} & $88.5 \pm 9.3$ & $80.1 \pm 4.3$ & 0.32 \\
\hline \multicolumn{2}{|l|}{ HR per $1 \mathrm{~min}$} & $71.8 \pm 8.1$ & $63.5 \pm 11.2$ & 0.04 \\
\hline \multicolumn{2}{|l|}{ BMI, $\mathrm{kg} / \mathrm{m}^{2}$} & $32.9 \pm 7.3$ & $29.1 \pm 0.6$ & 0.96 \\
\hline \multirow{2}{*}{$\begin{array}{l}\text { Waist circumfe- } \\
\text { rence, } \mathrm{cm}\end{array}$} & Men & $114.2 \pm 16.5$ & $118.7 \pm 9.2$ & 0.90 \\
\hline & Women & $108.0 \pm 14.1$ & $106.7 \pm 8.2$ & 0.56 \\
\hline \multirow{2}{*}{$\begin{array}{l}\text { Hip circumfe- } \\
\text { rence, } \mathrm{cm}\end{array}$} & Men & $113.0 \pm 12.2$ & $113.3 \pm 18.5$ & 0.33 \\
\hline & Women & $120.5 \pm 9.8$ & $119.8 \pm 11.4$ & 0.31 \\
\hline
\end{tabular}

Note: categorical parameters are given as the number of cases and the share, quantitative ones are given as $M \pm S D$ or as the median and the first - the third quartile; SBP - systolic blood pressure; DBP-diastolic blood pressure; HR - heart rate

Random effects: 1-factor «Behavioural Problems» and 2-factor combinations of TG and LDL cholesterol. Random effects all had significant positive covariances (Fig. 2). General characteristics of the model are presented in Fig. 3. We should note the high classification qualities of the constructed model: overall accuracy $-89.6 \%$; the accuracy of the «Insomnia» classification is $91.8 \%$ for «No insomnia signs» and $85.7 \%$ for «Insomnia» (Fig. 4).

\section{Fixed Effects}

Target:Insomnia

Reference Category:2.00

\begin{tabular}{|l|ccrr|}
\hline Source & F & df1 & df2 & Sig. \\
\hline Corrected Model $\boldsymbol{\nabla}$ & 7.335 & 4 & 110 & .000 \\
\hline Total_cholesterol & 8.037 & 1 & 110 & .005 \\
\hline Difficulty_initiating_sleep & 9.020 & 1 & 110 & .003 \\
\hline Difficulty_maintaining_sleep & 12.841 & 1 & 110 & .001 \\
\hline Concentration_on_sleep & 11.593 & 1 & 110 & .001 \\
\hline
\end{tabular}

Probability distribution:Multinomial

Link function:Generalized logit

Fig. 1. Model (fixed effects) 


\section{Random Effect Covariances}

Target: Insomnia

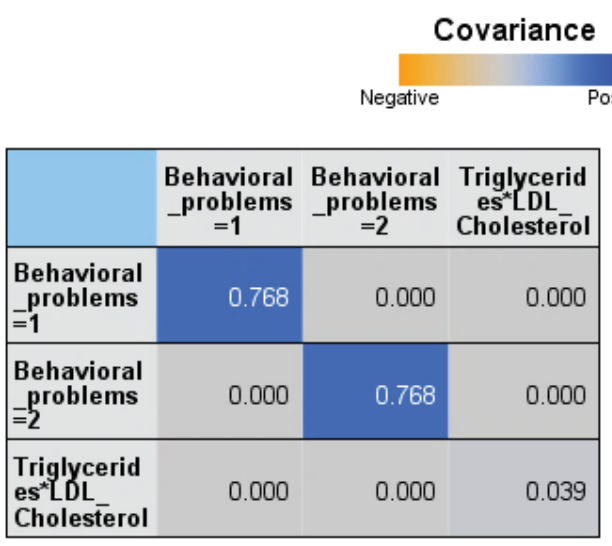

Fig. 2. Model (random effects)

Target: Insomnia

\begin{tabular}{|c|c|}
\hline Target & Insomnia \\
\hline Measurement Level & Nominal \\
\hline Probability Distribution & Multinomial \\
\hline Link Function & Generalized logit \\
\hline \multirow{2}{*}{ Information Criterion - } & 648.082 \\
\hline & 653.371 \\
\hline
\end{tabular}

Fig. 3. Overall characteristics of the model and information criteria

\section{Classification}

Target:Insomnia

Overall Percent Correct $=\mathbf{8 9 . 6} \%$

\begin{tabular}{|c|c|c|c|}
\hline \multirow{2}{*}{ Observed } & \multicolumn{2}{|l|}{ Predicted } & Row Perce \\
\hline & no Insomnia signs & Insomnia & $\square 100.00$ \\
\hline no Insomnia signs & $91.8 \%$ & $8.2 \%$ & $\begin{array}{l}60.00 \\
40.00\end{array}$ \\
\hline Insomnia & $14.3 \%$ & $85.7 \%$ & 0.00 \\
\hline
\end{tabular}

Fig. 4. Sensitivity and specificity of insomnia model

The t-test for independent groups confirms the significance of the difference in the mean values of total cholesterol and LDL at a level of $p<0.05$. The K-S test confirms the normality of total cholesterol and LDL at a level of $p<0.05$, because D-statistics for them are insignificant, 
and the W-test confirms the normality of total cholesterol only at the level of $p=0.052$ and HDL at the level of $p=0.09$. Therefore, let us additionally perform nonparametric tests for independent groups (Table 2).

Table 2

Blood lipid parameters in groups of patients with and without insomnia, $\mathrm{M} \pm \mathrm{SD}$

\begin{tabular}{llc}
\hline \multicolumn{1}{c}{ Cholesterol } & \multicolumn{2}{c}{ Insomnia } \\
\cline { 2 - 3 } & $\mathbf{1}(N=\mathbf{7 3})$ & $\mathbf{2}(N=\mathbf{4 2})$ \\
\hline TCS $\left(\mathrm{M} \pm \mathrm{SD}^{* *}\right), \mathrm{mmol} / \mathrm{l}$ & $5.17 \pm 1.02$ & $5.64 \pm 1.22^{*}$ \\
$\mathrm{TG}\left(\mathrm{M} \pm \mathrm{SD}^{* *}\right), \mathrm{mmol} / \mathrm{l}$ & $2.09 \pm 1.55$ & $1.86 \pm 1.43$ \\
$\mathrm{HDL}\left(\mathrm{M} \pm \mathrm{SD}^{* *}\right), \mathrm{mmol} / 1$ & $1.60 \pm 0.57$ & $1.31 \pm 0.29^{*}$ \\
LDL $\left(\mathrm{M} \pm \mathrm{SD}^{* *}\right), \mathrm{mmol} / 1$ & $2.94 \pm 0.97$ & $3.48 \pm 1.23^{*}$ \\
VLDL $\left(\mathrm{M} \pm \mathrm{SD}^{* *}\right), \mathrm{mmol} / 1$ & $0.95 \pm 0.73$ & $0.84 \pm 0.64$ \\
Glucose $\left(\mathrm{M} \pm \mathrm{SD}^{* *}\right), \mathrm{mmol} / \mathrm{l}$ & $6.37 \pm 2.34$ & $6.96 \pm 3.04$ \\
HbAlc $\left(\mathrm{M} \pm \mathrm{SD}^{* *}\right), \%$ & $6.34 \pm 0.92$ & $6.57 \pm 0.99$
\end{tabular}

${ }^{*} p<0.05$ comparison between patients with insomnia and without insomnia

The Wald-Wolfowitz test confirms that the data are obtained from a single population. The K-S 2-sample test and the Mann-Witney U-test confirm the significance of the difference in the values of total cholesterol and HDL at the level of $p<0.05$. Finally, the Kruskal-Wallis ANOVA and Median Test for multiple independent groups confirms the significance of the difference in the values of total cholesterol and HDL at the level of $p=0.01$ (Table 3).

Table 3

Odds ratio, confidence interval and significance for different insomnia signs

\begin{tabular}{lccc}
\hline \multicolumn{1}{c}{ History } & \multicolumn{3}{c}{ Insomnia } \\
\cline { 2 - 4 } & OR (odds ratio) & $\mathbf{9 5}$ \% CI & Significance \\
\hline Difficulty initiating sleep & 12.71 & $5.1-31.6$ & $p<0.0001$ \\
Difficulty maintaining sleep & 14.21 & $5.0-40.6$ & $p<0.0001$ \\
Concentration on sleep & 11.27 & $4.6-27.9$ & $p<0.0001$ \\
Behavioural problems & 2.82 & $1.1-6.9$ & $p<0.05$
\end{tabular}

\section{Discussion}

The unconditional role of night apnoea in the formation of cardiovascular risk, resistant hypertension, glucose metabolism disorders has been proven. That is why patients with night apnoea were excluded from this study.

When studying the relationship between sleep disorders and hypertension, most researchers have focused on sleep duration. It has been established that both short sleep, less than 6 hours, and sleep more than 8 hours are associated with the risk of developing hypertension, lipid metabolism disorders and increased BMI. The average age of study participants corresponded to 65.5 years, more than $60 \%$ of subjects suffered from hypertension. It was found that the relationship between sleep duration and HDL cholesterol level had the form of a U-curve. So, what low HDL cholesterol did patients with a sleep duration of less than 6 hours and more than 8 hours have. Those who slept less than 7 hours were found to have $31.1 \%$ higher HDL cholesterol levels than patients with shorter sleep duration [4]. It has been shown that individuals who sleep less than 6 hours have significantly lower HDL cholesterol in a data analysis of 162,121 men and women found a higher risk [6]. An association between short sleep (less than 5 hours) and low-density lipoprotein 
cholesterol and high triglycerides has been demonstrated for both men and women [12]. Another study, which included 2,705 patients, found no link between short sleep and HDL cholesterol [13]. But this study included patients with physiologically short sleep. In this case, the assessment of daytime drowsiness, or the use of insomnia criteria according to the International Classification of Sleep Disorders [14] is of particular importance.

Data on the effect of insomnia on lipid parameters are very limited [15-17]. No association has been established between insomnia and the development of dyslipidemia in persons not receiving sleeping pills [16]. A number of studies demonstrate the relationship between insomnia and the risk of high blood pressure, lower HDL cholesterol, increased waist circumference [18].

It was found that the reduction of insomnia in people with cardiovascular disease for 12 weeks was accompanied by a decrease in LDL cholesterol from 2.29 to $1.43 \mathrm{mmol} / \mathrm{l}$. But for the correction of insomnia, the expansion of physical activity was used, so it is not possible to associate these changes only with the correction of insomnia [2].

Study limitations. This study is not large and was conducted on a small sample of patients. Nevertheless, the data obtained will expand the view of scientists and doctors on the relationship and influence of sleep disorders and lipid metabolism.

Prospects for further research. Studying the effect of sleep disorders on metabolic parameters and lipid levels will allow the development of individualized therapies and improved correction of cardiovascular risk.

\section{Conclusion}

Thus, a relationship between total cholesterol, low-density lipoprotein cholesterol and the presence of insomnia has been established and proved by statistical model. The overall statistical model accuracy is $89.6 \%$ and statistical significance $p<0.005$. Accuracy of Insomnia prediction is $85.7 \%$ by level of total cholesterol (TCS) and patient interview data. This relationship is independent and does not depend on other random parameters.

\section{Conflict of interests}

The authors declare that they have no conflicts of interest.

\section{References}

[1] Manolis, T. A., Manolis, A. A., Apostolopoulos, E. J., Melita, H., Manolis, A. S. (2020). Cardiovascular Complications of Sleep Disorders: A Better Night's Sleep for a Healthier Heart / From Bench to Bedside. Current Vascular Pharmacology, 19 (2), 210-232. doi: http://doi.org/10.2174/1570161118666200325102411

[2] Rouleau, C. R., Toivonen, K., Aggarwal, S., Arena, R., Campbell, T. S. (2017). The association between insomnia symptoms and cardiovascular risk factors in patients who complete outpatient cardiac rehabilitation. Sleep Medicine, 32, $201-207$. doi: http://doi.org/10.1016/j.sleep.2017.01.005

[3] Lindberg, E., Janson, C., Johannessen, A., Svanes, C., Real, F. G., Malinovschi, A. et. al. (2020). Sleep time and sleep-related symptoms across two generations - results of the community-based RHINE and RHINESSA studies. Sleep Medicine, 69, 8-13. doi: http://doi.org/10.1016/j.sleep.2019.12.017

[4] Lin, P., Chang, K.-T., Lin, Y.-A., Tzeng, I.-S., Chuang, H.-H., Chen, J.-Y. (2017). Association between self-reported sleep duration and serum lipid profile in a middle-aged and elderly population in Taiwan: a community-based, cross-sectional study. BMJ Open, 7 (10), e015964. doi: http://doi.org/10.1136/bmjopen-2017-015964

[5] Buysse, D. J. (2013). Insomnia. JAMA, 309 (7), 706-716. doi: http://doi.org/10.1001/jama.2013.193

[6] Deng, H.-B., Tam, T., Zee, B. C.-Y., Chung, R. Y.-N., Su, X., Jin, L. et. al. (2017). Short Sleep Duration Increases Metabolic Impact in Healthy Adults: A Population-Based Cohort Study. Sleep, 40 (10). doi: http://doi.org/10.1093/sleep/zsx130

[7] Van der Wulp, M. Y. M., Verkade, H. J., Groen, A. K. (2013). Regulation of cholesterol homeostasis. Molecular and Cellular Endocrinology, 368 (1-2), 1-16. doi: http://doi.org/10.1016/j.mce.2012.06.007

[8] Kandeger, A., Egilmez, U., Sayin, A. A., Selvi, Y. (2018). The relationship between night eating symptoms and disordered eating attitudes via insomnia and chronotype differences. Psychiatry Research, 268, 354-357. doi: http://doi.org/10.1016/ j.psychres.2018.08.003

[9] Carter, J. R., Grimaldi, D., Fonkoue, I. T., Medalie, L., Mokhlesi, B., Van Cauter, E. (2018). Assessment of sympathetic neural activity in chronic insomnia: evidence for elevated cardiovascular risk. Sleep, 41 (6). doi: http://doi.org/10.1093/sleep/zsy048 
[10] Nestel, P. J., Khan, A. A., Straznicky, N. E., Mellett, N. A., Jayawardana, K., Mundra, P. A. et. al. (2017). Markers of sympathetic nervous system activity associate with complex plasma lipids in metabolic syndrome subjects. Atherosclerosis, 256, 21-28. doi: http://doi.org/10.1016/j.atherosclerosis.2016.11.032

[11] McCormack, T., Boffa, R. J., Jones, N. R., Carville, S., McManus, R. J. (2019). The 2018 ESC/ESH hypertension guideline and the 2019 NICE hypertension guideline, how and why they differ. European Heart Journal, 40 (42), 3456-3458. doi: http:// doi.org/10.1093/eurheartj/ehz681

[12] Okamura, T., Hashimoto, Y., Hamaguchi, M., Obora, A., Kojima, T., Fukui, M. (2019). Short Sleep Duration is a Risk of Incident Nonalcoholic Fatty Liver Disease: A Population-based Longitudinal Study. Journal of Gastrointestinal and Liver Diseases, 28 (1), 73-81. doi: http://doi.org/10.15403/jgld.2014.1121.281.alc

[13] Smiley, A., King, D., Harezlak, J., Dinh, P., Bidulescu, A. (2019). The association between sleep duration and lipid profiles: the NHANES 2013-2014. Journal of Diabetes \& Metabolic Disorders, 18 (2), 315-322. doi: http://doi.org/10.1007/ s40200-019-00415-0

[14] Sateia, M. J. (2014). International classification of sleep disorders-third edition: highlights and modifications. Chest, 146 (5), 1387-1394. doi: http://doi.org/10.1378/chest.14-0970

[15] Kruisbrink, M., Robertson, W., Ji, C., Miller, M. A., Geleijnse, J. M., Cappuccio, F. P. (2017). Association of sleep duration and quality with blood lipids: a systematic review and meta-analysis of prospective studies. BMJ Open, 7 (12), e018585. doi: http:// doi.org/10.1136/bmjopen-2017-018585

[16] Vozoris, N. T. (2016). Insomnia Symptoms Are Not Associated with Dyslipidemia: A Population-Based Study. Sleep, 39 (3), 551-558. doi: http://doi.org/10.5665/sleep.5524

[17] Zhan, Y., Zhang, F., Lu, L., Wang, J., Sun, Y., Ding, R. et. al. (2014). Prevalence of dyslipidemia and its association with insomnia in a community based population in China. BMC Public Health, 14 (1). doi: http://doi.org/10.1186/1471-2458-14-1050

[18] Syauqy, A., Hsu, C.-Y., Rau, H.-H., Kurniawan, A. L., Chao, J. C.-J. (2019). Association of Sleep Duration and Insomnia Symptoms with Components of Metabolic Syndrome and Inflammation in Middle-Aged and Older Adults with Metabolic Syndrome in Taiwan. Nutrients, 11 (8), 1848. doi: http://doi.org/10.3390/nu11081848 